le point sur..

\title{
Inde : un nouveau paysage éducatif
}

Padma M. Sarangapani

Traducteur : Sylvaine Herold

\section{(2) OpenEdition}

1 Journals

Édition électronique

URL : https://journals.openedition.org/ries/5877

DOI : 10.4000/ries.5877

ISSN : 2261-4265

Éditeur

France Education international

Édition imprimée

Date de publication : 1 septembre 2017

Pagination : 15-19

ISBN : 978-2-85420-615-9

ISSN : $1254-4590$

Référence électronique

Padma M. Sarangapani, «Inde : un nouveau paysage éducatif », Revue internationale d'éducation de Sèvres [En ligne], 75 | septembre 2017, mis en ligne le 01 septembre 2019, consulté le 25 juin 2021.

URL : http://journals.openedition.org/ries/5877 ; DOI : https://doi.org/10.4000/ries.5877

Ce document a été généré automatiquement le 25 juin 2021

(c) Tous droits réservés 
le point sur...

\title{
Inde : un nouveau paysage éducatif
}

\author{
Padma M. Sarangapani
}

Traduction : Sylvaine Herold

1 Le parti du peuple indien (Bharatiya Janata Party, BJP) ${ }^{1}$ a remporté les élections nationales de 2015 à une majorité écrasante, conduisant au pouvoir Narendra Modi et balayant toute opposition. Il n'existe actuellement pas de chef de file reconnu de l'opposition à la Chambre du peuple ${ }^{2}$. On s'attendait à ce que le nouveau gouvernement, de droite, annonce des changements et des initiatives en matière d'éducation, conformément à son idéologie et à sa source d'inspiration idéologique - le Rashtriya Sevak Sangh (RSS) ${ }^{3}$. Comme prévu, le gouvernement a annoncé la mise en œuvre d'un processus pour la formulation d'une nouvelle politique éducative (la précédente politique nationale datait de 1989).

2 Voici maintenant deux ans que ce processus pour une nouvelle politique éducative (New Education Policy: NEP) est en cours. Un comité de fonctionnaires à la retraite ne comprenant qu'un seul spécialiste d'éducation a mené une consultation nationale, recueillant l'opinion du peuple indien via des contributions en ligne et des réunions de consultation, qui proposaient treize thèmes de discussion pour l'enseignement scolaire et vingt pour l'enseignement supérieur. Selon le site Internet du ministère, 110623 des 244252 organismes villageois, 725 des 4027 municipalités, 2738 des 6620 blocs, 340 des 669 districts et 19 des 36 États ou territoires de l'Union ${ }^{4}$ ont pris part à ces consultations ou ont envoyé leurs contributions en ligne pour la nouvelle politique éducative. Il s'agit sans nul doute du processus consultatif le plus vaste jamais mené pour l'élaboration d'une politique éducative dans toute l'histoire de l'éducation moderne! On ne sait cependant pas clairement quelles contributions spécifiques ont été reçues de chacune de ces consultations - nous n'avons pu trouver sur le site ni résumé des contributions clés ni mention de la méthodologie utilisée pour consolider l'ensemble de ces données. Le comité responsable du développement de la nouvelle politique éducative a remis son rapport le 30 avril 2016 et l'on s'attendait à ce que la nouvelle politique soit annoncée dans les mois suivants. Mais, en juillet dernier, un nouveau comité a été constitué, afin de finaliser son élaboration. 
3 Cela ne veut pas dire pour autant que les choses en sont à un point de statu quo. Certaines évolutions n'ont pas besoin de proclamation politique en grande pompe et sont déjà à l'œuvre sur le terrain, dans l'enseignement scolaire et dans l'enseignement supérieur. Et, sans nul doute, ces évolutions de terrain importent plus que l'élaboration d'une politique, qui représente plus une déclaration d'intention et d'ambition qu'une pratique concrète. Dans cet article, nous évoquons des évolutions à l'œuvre dans trois domaines, révélatrices du climat actuel dans l'enseignement scolaire et supérieur indien.

\section{Liberté d'expression dans l'enseignement supérieur}

4 Après seulement quelques mois au pouvoir central, le BJP a été confronté à des troubles étudiants qui impliquaient des Dalits ${ }^{5}$ sur deux campus et des groupes étudiants de gauche sur plusieurs campus d'établissements d'enseignement supérieur d'élite. Des étudiants dalits, par ailleurs actifs dans les cercles d'études dalits et la vie politique du campus, en particulier en ce qui concerne la discrimination exercée par certains administrateurs ou professeurs de la caste supérieure, s'étaient heurtés à des membres de partis étudiants affiliés au BJP. L'escalade des affrontements sur les campus a finalement conduit à l'exclusion temporaire des étudiants dalits - suite à quoi l'un d'entre eux s'est donné la mort. Ailleurs, des groupes étudiants de gauche, qui organisaient des débats et critiquaient l'action du gouvernement en matière de "terrorisme" ou sa politique au Cachemire, ont fait l'objet d'actions de police en raison de leur prétendu caractère antinational. L'implication directe du ministère du développement des ressources humaines dans ces événements et ses directives aux vice-recteurs des universités représentent un niveau d'ingérence sans précédent dans l'autonomie des universités. Il semble être dans l'air du temps d'être intolérant vis-àvis des étudiants qui "gaspillent leur temps et les ressources de la nation" en s'impliquant dans des activités politiques et des débats, alors qu'ils devraient se consacrer à leurs études, à l'université et contribuer au développement de la nation. Les campus universitaires, qui ont de tout temps été des lieux importants d'expression d'une citoyenneté libérale, y compris via la dissidence, mais également pour la formation politique des futurs citoyens, via des discussions et des débats animés, ont désormais reçu le message qu'ils ne doivent plus encourager une telle liberté d'expression ni accueillir ou héberger des militants ou des penseurs politiques. Toute critique à l'encontre de l'action gouvernementale est considérée comme étant " antinationale ", limitant ainsi la liberté d'expression. On attend des étudiants qu'ils se concentrent sur l'excellence et qu'ils rendent compte, par leurs résultats, des ressources qui leur sont consacrées. Les étudiants et les facultés devraient en outre éviter de se mêler de politique et se consacrer aux matières universitaires. Un système de classement des universités a en outre été introduit et des réformes sont en cours, qui lient les ressources et le statut des universités à des mesures de performance.

\section{Le droit à l'éducation}

5 Le droit à l'éducation a été promulgué en Inde en 2009 et a fait l'objet d'importants débats, notamment en ce qui concerne: la clause exigeant des écoles privées non

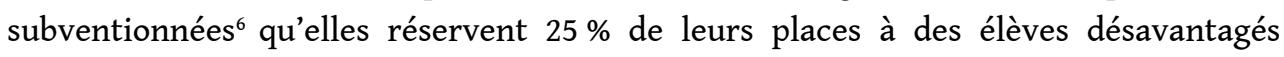


financièrement ; les clauses relatives à l'exigence de «non-redoublement $»^{7}$ des élèves (c'est-à-dire le passage automatique de niveau en niveau sans que l'élève soit mis en situation d'échec) et à l'évaluation continue et globale plutôt que par des examens annuels. La Cour suprême de l'Inde a statué en faveur de la constitutionalité de la clause des $25 \%$ en règle générale, mais elle en a exempté les écoles religieuses ou de minorités linguistiques. Dans les faits, cela a conduit à l'augmentation du nombre d'écoles privées non subventionnées revendiquant le statut d'école de minorité s'engouffrant dans la faille leur permettant de conserver leur clientèle exclusive! La politique de «non-redoublement» a par ailleurs fait l'objet de nombreuses critiques. Cette politique est déjà mise en œuvre dans les écoles gouvernementales et il est courant d'entendre les enseignants se plaindre qu'en raison de cette politique, les enfants n'ont plus peur de l'échec et n'étudient plus. Les directeurs d'écoles affirment, quant à eux, que les enseignants auraient commencé à négliger leur enseignement, compte tenu du fait que les élèves ne peuvent plus échouer. Ces critiques, conjuguées à la faiblesse générale des résultats d'apprentissage des élèves dans les écoles gouvernementales, ont conduit la politique de non-redoublement à être identifiée comme le principal problème à l'origine de la baisse de niveau. Et les écoles privées de se plaindre de conserve, arguant que cela aboutira également à la baisse des niveaux d'apprentissage dans leurs établissements. À l'origine, cette clause avait été intégrée dans la Loi sur le droit à l'éducation, afin de protéger les enfants de la stigmatisation liée à l'échec scolaire, en plaçant la responsabilité de l'éducation sur les écoles - mais sans trouver, semble-t-il, d'écho politique favorable. Aujourd'hui, la plupart des États indiens ont adopté des règlements types abrogeant la politique de non-redoublement et ils ont également promulgué le rétablissement des examens standardisés au niveau des classes 5 ( 11 ans) et 8 ( 14 ans), jugés nécessaires, notamment afin de garantir la responsabilisation des enseignants. La notion de responsabilité émerge actuellement comme un thème central - en particulier la responsabilité des enseignants pour l'apprentissage des élèves.

\section{Cadre curriculaire et manuels nationaux}

On s'attendait à ce que la révision du Cadre curriculaire national (National Curriculum Framework: NCF), élaboré en 2005, et des manuels figurent en bonne place parmi les priorités du nouveau gouvernement; et ce d'autant plus que le NCF 2005 avait été considéré comme une réaction visant à infléchir la "safranisation $»^{9}$ du curriculum et des manuels qui prévalait alors. Mais cela n'a pas été le cas. Le nouveau gouvernement a, dès le départ, totalement repris à son compte le NCF 2005, saluant la modernité des principes à partir desquels il avait été formulé. "Le comité recommande que les principes directeurs de la réforme curriculaire tels qu'énoncés dans le NCF 2005 soient considérés comme pertinents et activement mis en œuvre» (p. 106 du document de discussion de la NEP 2016). Le ministère a également mis un terme à la révision des documents curriculaires. Les manuels mis au point par le Conseil national pour la recherche et la formation pédagogiques (National Council of Educational Research and Training) ont été conservés, sans changement. Il semble que le ministère de l'éducation s'efforce d'apparaître comme moderne, tourné vers le développement et préoccupé par les faibles niveaux d'apprentissage, d'efficacité et de responsabilité dans le système scolaire gouvernemental, plutôt que d'avoir l'air influencé par le nationalisme Hindutva ${ }^{10}$ ou guidé par des intérêts idéologiques. Le seul sujet ayant fait l'objet d'une 
attention nationale est celui du yoga, promu comme matière d'enseignement sous l'intitulé "yoga pour la santé et le bien-être ». "Le yoga recevra une attention particulière. Des efforts seront faits pour que le yoga soit introduit dans l'ensemble des écoles. " (p. 100). L'inclusion de la pratique du yoga à l'école, promue par le MHRD, reçoit actuellement un puissant soutien. Mais, comme on pouvait s'y attendre, cela a suscité des inquiétudes quant à savoir si cette pratique devait être considérée comme la promotion effective de pratiques hindoues à l'école - car elle peut comprendre le chant du mantra «om» et la salutation au soleil sous la forme du "surya namaskar » ${ }^{11}$. Le MHRD lui-même a soigneusement évité toute référence aux liens entre religion et yoga, soulignant plutôt ses vertus, internationalement reconnues, pour le bien-être physique et mental. Face à la rumeur malveillante faisant état d'une fatwa lancée contre le yoga, le Darul-uloom Deoband, un séminaire islamique orthodoxe, a précisé qu'il n'y avait aucun problème avec cette "forme d'exercice ». Mais tout doute ou examen de ce qui est compris exactement sous l'appellation de " yoga » suscite immédiatement son lot de commentaires stridents et fallacieux en provenance des groupes d'extrême droite.

Dans certains des États dirigés par le BJP, au Rajasthan notamment, les manuels d'histoire ont été révisés pour intégrer une version Hindutva de la lutte pour la liberté de l'Inde, qui redéfinit radicalement les rôles attribués aux héros nationaux, y compris le Mahatma Gandhi, réduisant le premier Premier ministre de l'Inde, Jawaharlal Nehru, à une figure mineure, tandis que certains personnages Hindutva sont promus au rang de héros, comme c'est le cas de Veer Savarkar, un admirateur de Hitler ${ }^{12}$.

8 On assiste actuellement en Inde à la convergence entre une droite ancienne et le néolibéralisme. Dans ses perspectives générales de développement, l'État indien considère la dissension politique comme une perte de temps et de ressources quand, au contraire, tous les citoyens devraient être unis pour le progrès de la nation. Dans ce contexte, la liberté d'expression est considérée comme un luxe inutile et l'activité de ceux n'ayant pas à justifier de leur temps ni de leurs résultats. C'est en fait le système éducatif tout entier qui se fait actuellement « discipliner » en Inde, à travers ce régime de responsabilité, qui vise à instaurer plus d'efficacité et à limiter le gaspillage. L'État fédéral indien a également envoyé des forces de police sur les campus ou exclu des étudiants pour avoir « perturbé » la vie du campus. Après des décennies de progrès vers une pédagogie "centrée sur l'enfant ", inscrite dans la Loi sur le droit à l'éducation à travers la clause de non-redoublement, la tendance s'est désormais inversée et la peur de l'échec fait son retour comme outil de responsabilisation: des enseignants pour l'enseignement et des élèves pour l'apprentissage.

La perspective développementale néolibérale promeut la supervision étroite de l'enseignement pour une plus grande efficacité et la notion de responsabilité pour de meilleurs résultats. C'est cette approche qui a été adoptée au niveau central, parallèlement à la montée en puissance de l'agenda idéologique Hindutva dans les structures vernaculaires. Si une nouvelle politique éducative est attendue dans les prochains mois en Inde, le nouveau paysage éducatif est déjà bel et bien en train de prendre forme, mais sans le nécessaire soubassement de droits et de libertés fondamentaux. 


\section{NOTES}

1. Le Bharatiya Janata Party (BJP) est l'un des deux principaux partis politiques indiens. Créé en 1980, le BJP est un parti de droite nationaliste hindoue considéré comme l'aile politique du Rashtriya Swayamsevak Sangh (RSS). (Source : Wikipédia) (NdT)

2. Le Parlement de l'Inde est un parlement bicaméral, comprenant le Conseil des États (Rajya Sabha) et la Chambre du peuple (Lok Sabha). Le chef du parti majoritaire à la Lok Sabha est traditionnellement nommé Premier ministre de l'Inde. (Source : Wikipédia) (NdT)

3. Le Rashtriya Swayamsevak Sangh (RSS) ou " organisation patriotique nationale » est un groupe extrémiste nationaliste hindou de droite et paramilitaire qui, outre les considérations religieuses, propage une conception raciale du peuple indien. (Source : Wikipédia) (NdT)

4. L'Inde est une république fédérale composée de 29 États et 7 Territoires de l'Union, eux-mêmes divisés en districts, puis en tehsils portant différents noms selon les États (ici appelés " blocs »). Au niveau local, l'organisation administrative des zones rurales et des zones urbaines diffère : les zones rurales relèvent $\mathrm{du}$ système des panchayats (ici: organismes villageois) et les zones urbaines des municipalités. (Source : Wikipédia) (NdT)

5. «Dalit " est le nom générique donné à l'ancienne caste des «intouchables »; ces derniers bénéficient de dispositifs de discrimination positive dans les établissements d'éducation.

6. Il existe trois types d'écoles en Inde : les écoles du gouvernement (government schools), gérées et financées par le gouvernement de chaque État; les écoles privées aux frais de scolarité plus élevés, surtout destinées aux classes moyennes urbaines; et les écoles privées subventionnées par le gouvernement, pour réduire les frais de scolarité et les rendre accessibles aux enfants des familles pauvres. (Source: S. Herold et T. Mamode (2014), fiche documentaire sur l'Inde à l'occasion du colloque international « L'éducation en Asie », CIEP, 12-14 juin 2014 [https://goo.gl/ X9tSo9]). (NdT)

7. No detention en anglais. (NdT)

8. Accountability en anglais. (NdT)

9. Le safran est la couleur associée à l'hindouisme; la «safranisation » fait donc référence à l'influence hindoue.

10. L'Hindutva (ou «hindouïté ») désigne le courant idéologique qui sous-tend le nationalisme hindou offensif qui influence actuellement la société indienne et dont l'arrivée au pouvoir du BJP est l'une des manifestations. (Source : Guillaume Gandelin (2016) : «L'hindutva, aux origines du nationalisme hindou ", Asialyst, [https://goo.gl/42Tr1k]). (NdT)

11. Nom de la salutation au soleil en sanskrit translittéré. (NdT)

12. L'Inde a une structure fédérale et les États ont leurs propres commissions scolaires et leurs propres manuels. 
INDEX

Palabras claves : derecho a la educación, enseñanza superior, libro de texto, sistema educativo, curriculum

Keywords : right to education, higher education, textbooks, educational system, curriculum Index géographique : Inde

Mots-clés : droit à l'éducation, enseignement supérieur, manuel scolaire, système éducatif, curriculum

\section{AUTEURS}

\section{PADMA M. SARANGAPANI}

Padma M. Sarangapani, professeur d'éducation au Tata Institute of Social Science (Inde), dirige le Centre pour l'innovation et la recherche action en éducation du campus de Bombay. Membre du Conseil national pour la formation des enseignants depuis 2013, elle a également contribué à plusieurs instances au niveau national et régional. Elle a été rédacteur en chef de Contemporary Education Dialogue (2002-2014) et est membre du conseil scientifique international du British Journal of Sociology of Education, depuis 2011. Ses travaux portent principalement sur les enseignants, le curriculum et les théories pédagogiques. Courriel : psarangapani@hotmail.com 